\title{
A Highly Scalable Parallel Implementation of H.264
}

\author{
Arnaldo Azevedo ${ }^{1}$, Ben Juurlink ${ }^{1}$, Cor Meenderinck ${ }^{1}$, Andrei Terechko*5, \\ Jan Hoogerbrugge ${ }^{2}$, Mauricio Alvarez ${ }^{3}$, Alex Ramirez ${ }^{3,4}$, Mateo Valero ${ }^{3,4}$ \\ 1 Delft University of Technology, Delft, the Netherlands \\ \{Azevedo, Cor, Benj\}@ce.et.tudelft.nl \\ 2 NXP, Eindhoven, The Netherlands \\ jan.hoogerbrugge@nxp.com \\ 3 Technical University of Catalonia (UPC), Barcelona, Spain \\ $\{$ alvarez, mateo\}@ac.upc.edu \\ 4 Barcelona Supercomputing Center (BSC), Barcelona, Spain \\ alex.ramirez@bsc.es \\ ${ }^{5}$ Vector Fabrics, Eindhoven, Netherlands \\ andrei@vectorfabrics.com
}

\begin{abstract}
Developing parallel applications that can harness and efficiently use future many-core architectures is the key challenge for scalable computing systems. We contribute to this challenge by presenting a parallel implementation of H.264 that scales to a large number of cores. The algorithm exploits the fact that independent macroblocks (MBs) can be processed in parallel, but whereas a previous approach exploits only intra-frame MB-level parallelism, our algorithm exploits intra-frame as well as inter-frame MB-level parallelism. It is based on the observation that inter-frame dependencies have a limited spatial range. The algorithm has been implemented on a many-core architecture consisting of NXP TriMedia TM3270 embedded processors. This required to develop a subscription mechanism, where MBs are subscribed to the kick-off lists associated with the reference MBs. Extensive simulation results show that the implementation scales very well, achieving a speedup of more than 54 on a 64-core processor, in which case the previous approach achieves a speedup of only 23. Potential drawbacks of the 3D-Wave strategy are that the memory requirements increase since there can be many frames in flight, and that the frame latency might increase. Scheduling policies to address these drawbacks are also presented. The results show that these policies combat memory and latency issues with a negligible effect on the performance scalability. Results analyzing the impact of the memory latency, L1 cache size, and the synchronization and thread management overhead are also presented. Finally, we present performance requirements for entropy (CABAC) decoding.
\end{abstract}

\section{Introduction}

The demand for computational power increases continuously as the consumer market forecasts new applications such as Ultra High Definition (UHD) video [1],

Azevedo, A., Juurlink, B., Meenderinck, C., Terechko, A., Hoogerbrugge, J., Álvarez, M., Ramírez, A., Valero, M. A highly scalable parallel implementation of H.264. "Transactions on HiPEAC", 2011, vol. 4, p. 111-134. DOI 10.1007/978-3-642-24568-8_6

The final publication is available at: https://link.springer.com/chapter/10.1007/978-3-642-24568-8_6 
3D TV [2], and real-time High Definition (HD) video encoding. In the past this demand was mainly satisfied by increasing the clock frequency and by exploiting more instruction-level parallelism (ILP). Due to the inability to increase the clock frequency much further because of thermal constraints and because it is difficult to exploit more ILP, multicore architectures have appeared on the market.

This new paradigm relies on the existence of sufficient thread-level parallelism (TLP) to exploit the large number of cores. Techniques to extract TLP from applications will be crucial to the success of multicores. This work investigates the exploitation of the TLP available in an H.264 video decoder on an embedded multicore processor. H.264 was chosen due to its high computational demands, wide utilization, and development maturity and the lack of "mature" future applications. although a 64-core processor is not required to decode a Full High Definition (FHD) video in real-time. Real-time encoding remains a problem and decoding is part of encoding. Furthermore, emerging applications such as 3DTV are likely to be based on current video coding methods [2].

In previous works [3] [4] we have proposed the 3D-Wave parallelization strategy for H.264 video decoding. It has been shown that the 3D-Wave strategy potentially scales to a much larger number of cores than previous strategies. However, the results presented there are analytical, analyzing how many macroblocks (MBs) could be processed in parallel assuming infinite resources, no communication delay, infinite bandwidth, and a constant MB decoding time. In other words, our previous work is a limit study.

In this paper, we make the following contributions:

- We present an implementation of the 3D-Wave strategy on an embedded multicore consisting of up to 64 TM3270 processors. Implementing the 3DWave turned out to be quite challenging. It required to dynamically identify inter-frame MB dependencies and handle their thread synchronization, in addition to intra-frame dependencies and synchronization. This led to the development of a subscription mechanism where MBs subscribe themselves to a so-called Kick-off List (KoL) associated with the MBs they depend on. Only if these MBs have been processed, processing of the dependent MBs can be resumed.

- A potential drawback of the 3D-Wave strategy is that the latency may become unbounded because many frames will be decoded simultaneously. A policy is presented that gives priority to the oldest frame so that newer frames are only decoded when there are idle cores.

- Another potential drawback of the 3D-Wave strategy is that the memory requirements might increase because of large number of frames in flight. To overcome this drawback we present a frame scheduling policy to control the number of frames in flight.

- We analyze the impact of the memory latency and the L1 cache size on the scalability and performance of the 3D-Wave strategy.

- The experimental platform features hardware support for thread management and synchronization, making it relatively light weight to submit/retrieve a task to/from the task pool. We analyze the importance of this hardware support by artificially increasing the time it takes to submit/retrieve a task. 
- The 3D-Wave focuses on the MB decoding part of the H.264 decoding and assumes an accelerator for entropy decoding. We analyze the performance requirements of the entropy decoding accelerator not to harm the 3D-Wave scalability.

Parallel implementations of H.264 decoding and encoding have been described in several papers. Rodriguez et al. [5] implemented an H.264 encoder using Group of Pictures (GOP)- (and slice-) level parallelism on a cluster of workstations using MPI. Although real-time operation can be achieved with such an approach, the latency is very high.

Chen et al. [6] presented a parallel implementation that decodes several B frames in parallel. However, even though uncommon, the H.264 standard allows to use B frames as reference frames, in which case they cannot be decoded in parallel. Moreover, usually there are no more than 2 or $3 \mathrm{~B}$ frames between $\mathrm{P}$ frames. This limits the scalability to a few threads. The 3D-Wave strategy dynamically detects dependencies and automatically exploits the parallelism if $B$ frames are not used as reference frames.

MB-level parallelism has been exploited in previous work. Van der Tol et al. [7] presented the exploitation of intra-frame MB-level parallelism and suggested to combine it with frame-level parallelism. If frame-level parallelism can be exploited is determined statically by the length of the motion vectors, while in our approach it is determined dynamically.

Chen et al. [6] also presented MB-level parallelism combined with frame-level parallelism to parallelize H.264 encoding. In their work, however, the exploitation of frame-level parallelism is limited to two consecutive frames and independent MBs are identified statically. This requires that the encoder limits the motion vector length. The scalability of the implementation is analyzed on a quad-core processor with Hyper-Threading Technology. In our work independent MBs are identified dynamically and we present results for up to 64 cores.

This paper is organized as follows. Section 2 provides an overview of MB parallelization technique for H.264 video decoding and the 3D-Wave technique. Section 3 presents the simulation environment and the experimental methodology to evaluate the 3D-Wave implementation. In Section 4 the implementation of the 3D-Wave on the embedded many-core is detailed and it introduces a frame scheduling policy to limit the number of frames in flight and describes a priority policy to reduce latency. Extensive simulation results, analyzing the scalability and performance of the baseline 3D-Wave, the frame scheduling and frame priority policies, as well as the impacts of the memory latency, L1 cache size, parallelization overhead, and entropy decoding, are presented in Section 5. Conclusions are drawn in Section 6.

\section{Thread-level parallelism in $\mathrm{H} .264$ video decoding}

Currently, H.264 [8] is one of the best video coding standard, in terms of compression and quality [9]. It has a compression improvement of over two times compared to previous standards such as MPEG-4 ASP, H.262/MPEG-2, etc. 
The H.264 standard was designed to serve a broad range of application domains ranging from low to high bitrates, from low to high resolutions, and a variety of networks and systems, e.g., internet streams, mobile streams, disc storage, and broadcast.

The coding efficiency gains of advanced video codecs such as H.264 come at the price of increased computational requirements. The computing power demand increases also with the shift towards high definition resolutions. As a result, current high performance uniprocessor architectures are not capable of providing the performance required for real-time processing $[10,11]$. Therefore, it is necessary to exploit parallelism. The H.264 codec can be parallelized either by a task-level or data-level decomposition.

In a task-level decomposition the functional partitions of the application such as vector prediction, motion compensation, and deblocking filter are assigned to different processors. Scalability is a problem because it is limited to the number of tasks, which typically is small. In a data-level decomposition the work (data) is divided into smaller parts and each part is assigned to a different processor. Each processor runs the same program but on different (multiple) data elements (SPMD). In H.264 data decomposition can be applied to different levels of the data structure. Only MB-level parallelism is described in this work; a discussion of the other levels can be found in [4].

In H.264, the motion vector prediction, intra prediction, and the deblocking filter kernels use data from neighboring MBs defining the dependencies shown in Figure 1. Processing MBs in a diagonal wavefront manner satisfies all the dependencies and allows to exploit parallelism between MBs. We refer to this parallelization technique as $2 \mathrm{D}$-Wave, to distinguish it from the 3D-Wave proposed in [4] and for which implementation results are presented in this work.

\begin{tabular}{|c|c|c|c|c|c|}
\hline $\begin{array}{c}\text { MB }(0,0) \\
\text { T1 }\end{array}$ & $\begin{array}{c}\text { MB (1,0) } \\
\text { T2 }\end{array}$ & $\begin{array}{c}\text { MB }(2,0) \\
\text { T3 }\end{array}$ & $\begin{array}{c}\text { MB }(3,0) \\
\text { T4 }\end{array}$ & $\begin{array}{c}\text { MB }(4,0) \\
\text { T5 }\end{array}$ & MBs processed \\
\hline $\begin{array}{c}\text { MB }(0,1) \\
\text { T3 }\end{array}$ & $\begin{array}{c}\text { MB }(1,1) \\
\text { T4 }\end{array}$ & $\begin{array}{c}\text { MB }(2,1) \\
\text { T5 }\end{array}$ & $\begin{array}{c}\mathrm{MB}(3,1) \\
\mathrm{T} 6\end{array}$ & $\mathrm{M}_{\mathrm{T}}$ & MBs in flight \\
\hline $\begin{array}{c}\text { MB }(0,2) \\
\text { T5 }\end{array}$ & $\begin{array}{l}\mathrm{MB}(1,2) \\
\mathrm{T} 6\end{array}$ & $\begin{array}{c}\overrightarrow{\mathrm{M}} \mathrm{B}(2,2) \\
\mathrm{T} 7\end{array}$ & $\begin{array}{c}\text { MB }(3,2) \\
\text { T8 }\end{array}$ & $\begin{array}{c}\text { MB }(4,2) \\
\text { T9 }\end{array}$ & \\
\hline $\begin{array}{c}\text { Mí }(0,3) \\
\text { T7 }\end{array}$ & $\begin{array}{c}\text { MB }(1,3) \\
\text { T8 }\end{array}$ & $\begin{array}{c}\text { MB }(2,3) \\
\text { T9 }\end{array}$ & $\begin{array}{c}\text { MB }(3,3) \\
\text { T10 }\end{array}$ & $\begin{array}{c}\mathrm{MB}(4,3) \\
\mathrm{T} 11\end{array}$ & \\
\hline $\begin{array}{c}\text { MB }(0,4) \\
\text { T9 }\end{array}$ & $\begin{array}{c}\text { MB }(1,4) \\
\text { T10 }\end{array}$ & $\begin{array}{c}\text { MB }(2,4) \\
\text { T11 }\end{array}$ & $\begin{array}{c}\text { MB }(3,4) \\
\text { T12 }\end{array}$ & $\begin{array}{c}\text { MB }(\mathbf{4 , 4}) \\
\text { T13 }\end{array}$ & \\
\hline
\end{tabular}

Fig. 1. 2D-Wave approach for exploiting MB parallelism. The arrows indicate dependencies.

Figure 1 illustrates the 2D-Wave for an image of $5 \times 5$ MBs $(80 \times 80$ pixels). At time slot $\mathrm{T} 7$ three independent MBs can be processed: $\operatorname{MB}(4,1), \operatorname{MB}(2,2)$, and $\operatorname{MB}(0,3)$. The number of independent MBs varies over time. At the start it increases with one MB every two time slots, then stabilizes at its maximum, 
and finally decreases at the same rate it increased. For a low resolution like QCIF there are at most 6 independent MBs during 4 time slots. For Full High Definition $(1920 \times 1088)$ there are 60 independent MBs during 9 time slots.

MB-level parallelism has several advantages over other H.264 parallelization schemes. First, this scheme can have good scalability, since the number of independent MBs increases with the resolution of the image. Second, it is possible to achieve good load balancing if dynamic scheduling is used.

MB-level parallelism also has some disadvantages, however. The first is that entropy decoding can only be parallelized using data-level decomposition at slicelevel, since the lowest level of data that can be parsed from the bitstream are slices. Only after entropy decoding has been performed the parallel processing of MBs can start. This disadvantage can be overcome by using special purpose instructions or hardware accelerators for entropy decoding. The second disadvantage is that the number of independent MBs is low at the start and at the end of decoding a frame. Therefore, it is not possible to sustain a certain processing rate during frame decoding.

The 2D-Wave technique, however, does not scale scales to future many-core architectures containing 100 cores or more, unless extremely high resolution frames are used. We have proposed [4] a parallelization strategy that combines intra-frame MB-level parallelism with inter-frame MB-level parallelism and which reveals the large amount of TLP required to harness and effectively use future many-core CMPs. The key points are described below.

In H.264 decoding there is only an inter-frame dependency in the Motion Compensation module. When the reference area has been decoded, it can be used by the referencing frame. Thus it is not necessary to wait until a frame is completely decoded before starting to decode the next frame. The decoding process of the next frame can start after the reference areas of the reference frames are decoded. Figure 2 illustrates this strategy called the 3D-Wave.

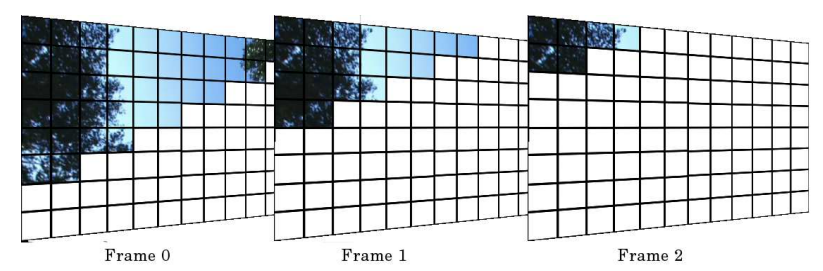

Fig. 2. 3D-Wave strategy: frames can be decoded in parallel because inter-frame dependencies have a limited spatial range.

In our previous study the FFMPEG H.264 decoder [4] was modified to analyze the available parallelism for real movies. The experiments did not consider any practical or implementation issues, but explored the limits to the parallelism available in the application. The results show that the number of parallel MBs exhibited by the 3D-Wave ranges from 1202 to 1944 MBs for SD resolution 
$(720 \times 576)$, from 2807 to $4579 \mathrm{MBs}$ for HD $(1280 \times 720)$, and from 4851 to 9169 MBs for FHD $(1920 \times 1088)$. To sustain this amount of parallelism, the number of frames in flight ranges from 93 to 304 depending on the input sequence and the resolution. So, theoretically, the parallelism available on 3D-Wave technique is huge. There are many factors in real systems, however, such as the memory hierarchy and bandwidth, that could limit its scalability. In the next sections the approach to implement the 3D-Wave and exploit this parallelism on an embedded manycore system is presented.

\section{Experimental methodology}

In this section the tools and methodology to implement and evaluate the 3DWave technique are detailed. Components of the many-core system simulator used to evaluate the technique are also presented.

An NXP proprietary simulator based on SystemC is used to run the application and collect performance data. Computations on the cores are modeled cycle-accurate. The memory system is modeled using average transfer times with channel and bank contention. When channel or bank contention is detected, the traffic latency is increased. NoC contention is supported. The simulator is capable of simulating systems with up to 64 TM3270 cores with shared memory and its cache coherence protocols. The operating system is not simulated.

The TM3270 [12] is a VLIW-based media-processor based on the Trimedia architecture. It addresses the requirements of multi-standard video processing at standard resolution and the associated audio processing requirements for the consumer market. The architecture supports VLIW instructions with five guarded issue slots. The pipeline depth varies from 7 to 12 stages. Address and data words are 32 bits wide. the unified register file has 12832 -bit registers. $2 \times 16$-bit and $4 \times 8$-bit SIMD instruction are supported. The TM3270 processor can run at up to $350 \mathrm{MHz}$, but in this work the clock frequency is set to $300 \mathrm{MHz}$. To produce code for the TM3270 the state-of-the-art highly optimizing NXP TriMedia $\mathrm{C} / \mathrm{C}++$ compiler version 5.1 is used.

The modeled system features a shared memory using MESI cache coherence. Each core has its own L1 data cache and can copy data from other L1 caches through 4 channels. The $64 \mathrm{Kbyte} \mathrm{L} 1$ data cache has 64 -byte lines and is 4 -way set-associative with LRU replacement and write allocate. The instruction cache is not modeled. The cores share a distributed L2 cache with 8 banks and an average access time of 40 cycles. The average access time takes into account L2 hits, misses, and interconnect delays. L2 bank contention is modeled so two cores cannot access the same bank simultaneously.

The multi-core programming model follows the task pool model. A Task Pool (TP) library implements submissions and requests of tasks to/from the task pool, synchronization mechanisms, and the task pool itself. In this model there is a main core and the other cores of the system act as slaves. Each slave runs a thread by requesting a task from the TP, executing it, and requesting 
another task. The task execution overhead is low. The time to request a task is less than $2 \%$ of the MB decoding time.

The experiments focus on the baseline profile of the H.264 standard. This profile only supports I and P frames and every frame can be used as a reference frame. This feature prevents the exploitation of frame-level parallelization techniques such as the one described in [6]. However, this profile highlights the advantages of the 3D-Wave, since the scalability gains come purely from the application of the 3D-Wave technique. Encoding was done with the X264 encoder [13] using the following options: no B-frames, at most 16 reference frames, weighted prediction, hexagonal motion estimation algorithm with a maximum search range of 24, and one slice per frame. The experiments use all four videos from the HD-VideoBench [14], Blue_Sky, Rush_Hour, Pedestrian, and Riverbed, in the three available resolutions, SD, HD and FHD.

The 3D-Wave technique focuses on the TLP available in the MB processing kernels of the decoder. The entropy decoder is known to be difficult to parallelize. To avoid the influence of the entropy decoder, its output has been buffered and its decoding time is not taken into account. Although not the main target, the 3D-Wave also eases the entropy decoding challenge. Since entropy decoding dependencies do not cross slice/frame borders, multiple entropy decoders can be used. We analyze the performance requirements of an entropy decoder accelerator on Section 5.7.

\section{Implementation}

In our previous work we used the FFmpeg decoder, but since we are using the Trimedia simulator for this implementation, we use the NXP H.264 decoder. The 2D-Wave parallelization strategy has already been implemented in this decoder [15], making it a perfect starting point for the implementation of the 3D-Wave. The NXP H.264 decoder is highly optimized, including both machinedependent optimizations (e.g. SIMD operations) and machine-independent optimizations (e.g. code restructuring).

The 3D-Wave implementation serves as a proof of concept thus the implementation of all features of H.264 is not necessary. Intra prediction inputs are deblock filtered samples instead of unfiltered samples as specified in the standard. This does not add visual artifacts to the decoded frames or change the MB dependencies.

This section details the 2D-Wave implementation used as the starting point, the 3D-Wave implementation, and the frame scheduling and priority policies.

\subsection{D-Wave implementation}

The MB processing tasks are divided in four kernels: vector prediction (VP), picture prediction (PP), deblocking info (DI), and deblocking filter (DF). VP calculates the motion vectors (MVs) based on the predicted motion vectors of the neighbor MBs and the differential motion vector present in the bitstream. PP 
performs the reconstruction of the MB based on neighboring pixel information (Intra Prediction) or on reference frame areas (Motion Compensation). Inverse quantization and the inverse DCT are also part of this kernel. DI calculates the strength of the DF based on MB data, such as the MBs type and MVs. DF smoothes block edges to reduce blocking artifacts.

The 2D-Wave is implemented per kernel. By this we mean that first VP is performed for all MBs in a frame, then PP for all MBs, etc. Each kernel is parallelized as follows. Figure 1 shows that within a frame each MB depends on at most four MBs. These dependencies are covered by the dependencies from the left $\mathrm{MB}$ to the current $\mathrm{MB}$ and from the upper-right $\mathrm{MB}$ to the current $\mathrm{MB}$, i.e., if these dependencies are satisfied then all dependencies are satisfied. Therefore, each MB is associated with a reference count between 0 and 2 representing the number of MBs it depends on. For example, the upper-left MB has a reference count of 0 , the other MBs at the top edge have a reference count of 1 , and so do the other MBs at the left edge. When a MB has been processed, the reference counts of the MBs that depend on it are decreased. When one of these counts reaches zero, a thread that will process the associated MB is submitted to the TP. Figure 3 depicts pseudo C-code for deblocking a frame and for deblocking a MB.

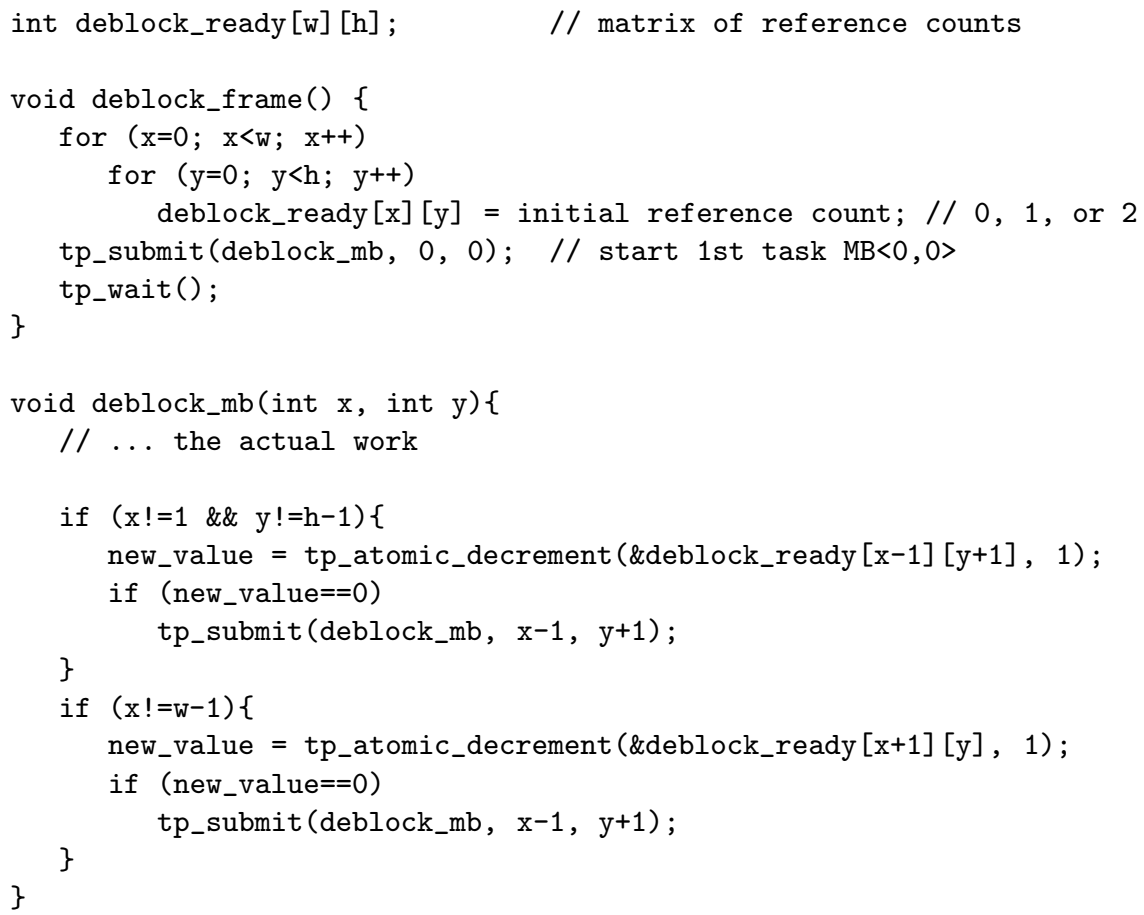

Fig. 3. Pseudo-code for deblocking a frame and a MB. 
When a core loads a MB in its cache, it also fetches neighboring MBs. Therefore, locality can be improved if the same core also processes the right MB. To increase locality and reduce task submission and acquisition overhead, the 2DWave implementation features an optimization called tail submit. After the MB is processed, the reference counts of the $\mathrm{MB}$ candidates are checked. If both $\mathrm{MB}$ candidates are ready to execute, the core processes the right $\mathrm{MB}$ and submits the other one to the task pool. If only one MB is ready, the core starts its processing without submitting or acquiring tasks to/from the TP. In case there is no neighboring $\mathrm{MB}$ ready to be processed, the task finishes and the core request another one from the TP. Figure 4 depicts pseudo-code for MB decoding after the tail submit optimization has been performed.

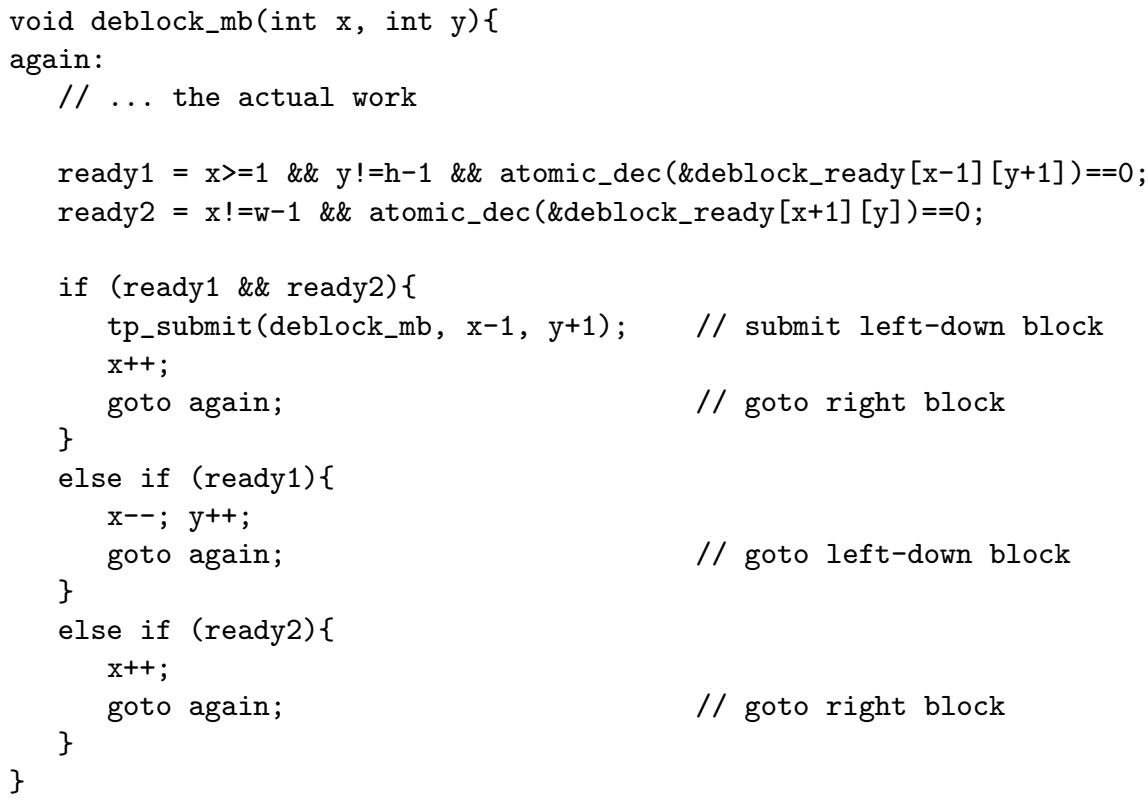

Fig. 4. Tail submit.

\subsection{D-Wave implementation}

In this section the 3D-Wave implementation is described. First we note that the original structure of the decoder is not suitable for the 3D-Wave strategy, because inter-frame dependencies are satisfied only after the DF is applied. To implement the 3D-Wave, it is necessary to develop a version in which the kernels are applied on a MB basis rather than on a slice/frame basis. In other words, we have a function decode_mb that applies each kernel to a MB. 
Since the 3D-Wave implementation decodes multiple frames concurrently, modifications to the Reference Frame Buffer (RFB) are required. The RFB stores the decoded frames that are going to be used as reference. As it can serve only one frame in flight, the 3D-Wave would require multiple RFBs. In this proof of concept implementation, the RFB was modified such that a single instance can serve all frames in flight. In the new RFB all the decoded frames are stored. The mapping of the reference frame index to RFB index was changed accordingly.

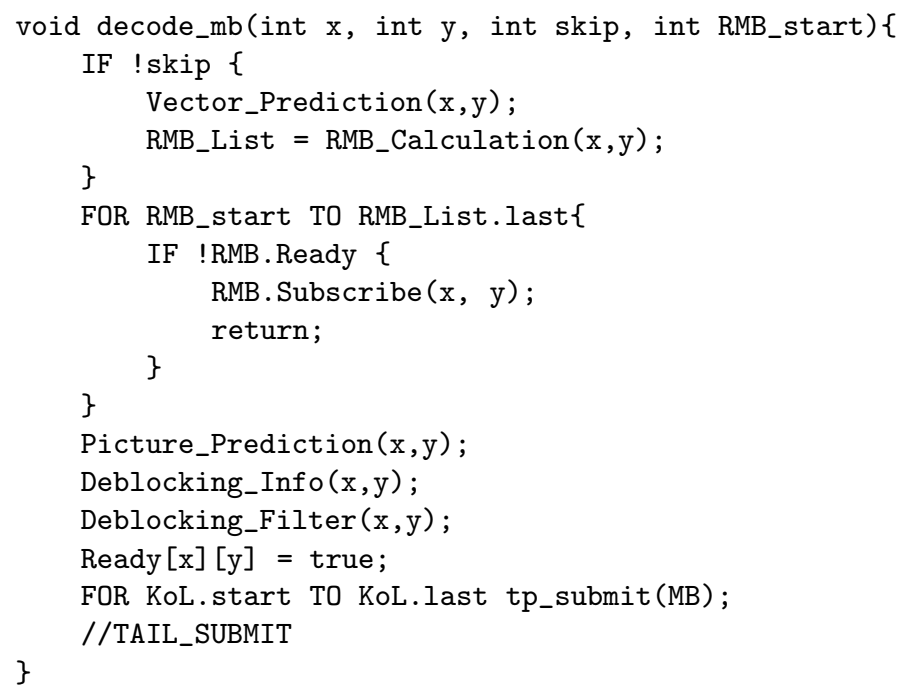

Fig. 5. Pseudo-code for 3D-Wave.

Figure 5 depicts pseudo-code for the decode_mb function. It relies on the ability to test if the reference MBs (RMBs) of the current MB have already been decoded or not. The RMB is defined as the MB in the bottom right corner of the reference area, including the extra samples for fractional motion compensation. To be able to test this, first the RMBs have to be calculated. If an RMB has not been processed yet, a method is needed to resume the execution of this MB after the RMB is ready.

The RMBs can only be calculated after motion vector prediction, which also defines the reference frames. Each MB can be partitioned in up to four $8 \times 8$ pixel areas and each one of them can be partitioned in up to four $4 \times 4$ pixel blocks The $4 \times 4$ blocks in an $8 \times 8$ partition share the reference frame. With the MVs and reference frames information, it is possible to calculate the RMB of each MB partition. This is done by adding the MV, the size of the partition, the position of the current $\mathrm{MB}$, and the additional area for fractional motion compensation and by dividing the result by 16 , the size of the MB. The RMB results of each partition is added to a list associated with the MB data structure, called the 
RMB-list. To reduce the number of RMBs to be tested, the reference frame of each RMB is checked. If two RMBs are in the same reference frame, only the one with the larger 2D-Wave decoding order (see Figure 1) is added to the list.

The first time decode_mb is called for a specific MB it is called with the parameter skip set to false and RMB_start set to 0 . If the decoding of this MB is resumed, it is called with the parameter skip set to true. Also RMB_start carries the position of the MB in the RMB-list to be tested next.

Once the RMB-list of the current MB is computed, it is verified if each RMB in the list has already been decoded or not. Each frame is associated with a MB ready matrix, similar to the deblock_ready matrix in Figure 3. The corresponding $\mathrm{MB}$ position in the ready matrix associated with the reference frame is atomically checked. If all RMBs are decoded, the decoding of this MB can continue.

To handle the cases where a RMB is not ready, a RMB subscription technique has been developed. The technique was motivated by the specifics of the TP library, such as low thread creation overhead and no sleep/wake up capabilities. Each MB data structure has a second list called the Kick-off List (KoL) that contains the parameters of the MBs subscribed to this RMB. When a RMB test fails, the current MB subscribes itself to the KoL of the RMB and finishes its execution. Each MB, after finishing its processing, indicates that it is ready in the ready matrix and verifies its KoL. A new task is submitted to the TP for each MB in the KoL.

The subscription process is repeated until all RMBs are ready. Finally, the intra-frame MBs that depend on this MB are submitted to the TP using tail submit, identical to Figure 4.

\subsection{Frame scheduling policy}

To achieve the highest speedup, all frames of the sequence are scheduled to run as soon as their dependencies are met. However, this can lead to a large number of frames in flight and large memory requirements, since every frame must be kept in memory. Mostly it is not necessary to decode a frame as soon as possible to keep all cores busy. A frame scheduling technique was developed to keep the working set to its minimum.

Frame scheduling uses the RMB subscription mechanism to define the moment when the processing of the next frame should be started. The first MB of the next frame can be subscribed to start after a specific MB of the current frame. With this simple mechanism it is possible to control the number of frames in flight. Adjusting the number of frames in flight is done by selecting an earlier or later $\mathrm{MB}$ with which the first $\mathrm{MB}$ of the next frame will be subscribed.

\subsection{Task priorities}

Latency is an important characteristic of video decoding systems. The frame scheduling policy described in the previous section reduces the frame latency, 
since the next frame is scheduled only when a part of the current frame has been decoded. However, when a new frame is scheduled to be decoded, the available cores are distributed equally among the frames in flight. A priority mechanism was added to the TP library in order to reduce the frame latency even further.

The TP library was modified to support two levels of priority. An extra task buffer was implemented to store high priority tasks. When the TP receives a task request, it first checks if there is a task in the high priority buffer. If so this task is selected, otherwise a task in the low priority buffer is selected. With this simple mechanism it is possible to give priority to the tasks belonging to the frame "next in line". Before submitting a new task the process checks if its frame is the frame "next in line". If so the task is submitted with high priority. Otherwise it is submitted with low priority. This mechanism does not lead to starvation because if there is not sufficient parallelism in the frame "next in line" the low priority tasks are selected.

\section{Experimental results}

In this section the experimental results are presented. The results include the scalability results of the 3D-Wave, the impact on the memory and bandwidth requirements, results of the frame scheduling and priority policies, and the influence of memory latency and the L1 data cache size on scalability and performance. The experiments were carried out according to the methodology described in Section 3. To evaluate the 3D-Wave technique, one second (25 frames) of each sequence was decoded using the enhanced NXP decoder. Longer sequences could not be simulated due to simulator constraints. The four sequences of the HD-VideoBench using three resolutions were evaluated. Due to space limitations only the results for the Rush_Hour sequence are presented which are close to the average. The results for the other sequences vary less than $5 \%$.

\subsection{Scalability}

The scalability results are for 1 to 64 cores. More cores could not be simulated due to limitations of the simulator. Figs. 6(a), 6(b), and 6(c) depict the 3D-Wave scalability on $p$ processors $\left(T_{3 D(1)} / T_{3 D(p)}\right) 2$ D-Wave scalability $\left(T_{2 D(1)} / T_{2 D(p)}\right)$, and $3 \mathrm{D}$-Wave versus $2 \mathrm{D}$-Wave on a single core $\left(T_{2 D(1)} / T_{3 D(p)}\right.$, labeled as $3 \mathrm{D}$ vs 2D. On a single core, 2D-Wave can decode $39 \mathrm{SD}, 18 \mathrm{HD}$, and 8 FHD frames per second.

On a single core the 3D-Wave implementation takes $8 \%$ more time than the 2D-Wave implementation due to administrative overhead. The 3D-Wave implementation scales almost perfectly up to 8 cores, while the 2D-Wave implementation incurs a $11 \%$ efficiency drop even for 2 cores due to the following reason. The tail submit optimization assigns MBs to cores per line. At the end of a frame, when a core finishes its line and there is no other line to be decoded, in the 2D-Wave the core remains idle until all cores have finished their line. If the 


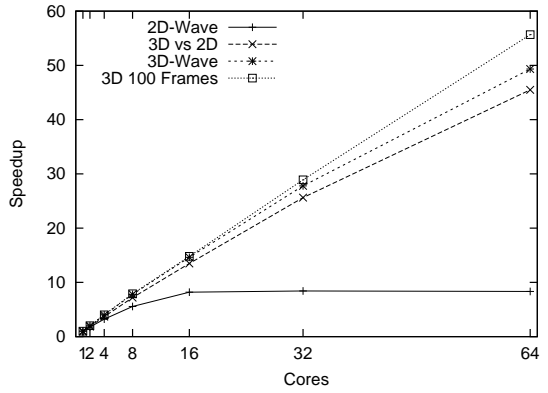

(a) SD.

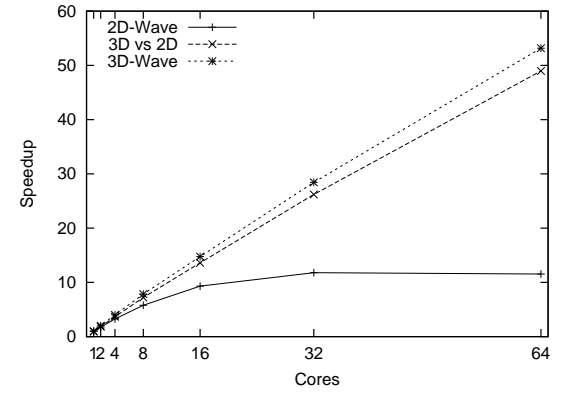

(b) HD.

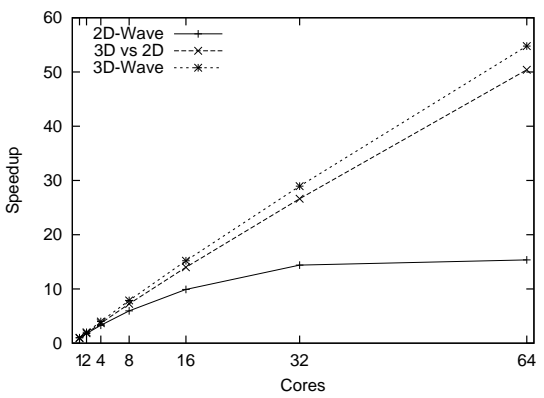

(c) FHD.

Fig. 6. 2D-Wave and 3D-Wave speedups in a 25-frame sequence Rush_Hour for different resolutions. 
last line happens to be slow the other cores wait for a long time and the core utilization is low. In the 3D-Wave, cores that finish their line, while there is no new line to be decoded, will be assigned a line of the next frame. Therefore, the core utilization as well as the scalability efficiency of the 3D-Wave is higher. Another advantage of the 3D-Wave over 2D-Wave is that it increases the efficiency of the Tail Submit optimization. In the 2D-Wave the low available parallelism makes the cores stall more due to unsolved intra-frame dependencies. In the 3D-Wave, the available parallelism is much larger which increases the distance between MB being decoded, minimizing intra-frame dependency stalls.

For SD sequences, the 2D-Wave technique saturates at 16 cores, with a speedup of only 8. This happens because of the limited amount of MB parallelism inside the frame and the dominant ramp up and ramp down of the availability of parallel MBs. The 3D-Wave technique for the same resolution continuously scales up to 64 cores, with a parallelization efficiency of almost $80 \%$. For the FHD sequence, the saturation of the 2D-Wave occurs at 32 cores while the $3 \mathrm{D}$-Wave continuously scales up to 64 cores with a parallelization efficiency of $85 \%$.

The scalability results of the 3D-Wave implementation in increase slightly for higher resolutions. On the other hand, the 2D-Wave implementation achieves higher speedups for higher resolutions since the MB-level parallelism inside a frame increases. However, it would take an extremely large resolution for the 2D-Wave to leverage 64 cores, and the 3D-Wave implementation would still be more efficient.

The drop in scalability efficiency of the 3D-Wave for larger number of cores has two reasons. First, cache trashing occurs for large number of cores, leading to a large number of memory stalls. It will be show in the next section. Second, at the start and at the end of a sequence, not all cores can be used because little parallelism is available. The more cores are used, the more cycles are wasted during these two periods. It would be negligible in a real sequence with many frames.

Simulations with FHD sequences with more than 25 frames are not possible because a simulator limitation. Fig. 6(a) includes the scalability result for 100 SD frames from Rush Hour.

For 64 cores the scalability grow from 49.32 to 55.67 when increasing from 25 to 100 frames. The effects of ramp up and ramp down times are minimized on the scalability results when more frames are used. On this case, the scalability results are closer to the results that would be achieved in a real life situation.

\subsection{Frame scheduling and priority}

In this section, experimental results for frame scheduling and frame priority are presented. The methodologies effectiveness are presented first, then the impact of these methodologies on the 3D-Wave efficiency.

Figure 7(a) presents the results of the frame scheduling technique applied to the FHD Rush_Hour sequence using a 16-core system. This figure presents the number of MBs processed per $m s$. It also shows to which frame these MBs belong. In this particular case, the subscribe MB chosen is the last $\mathrm{MB}$ on the 
line that is at $1 / 3 \mathrm{rd}$ of the frame. For this configuration there are 3 frames in flight. In the current state of development, the selection of the subscribe MB must be done statically by the programmer. A methodology to dynamically fire new frames based on core utilization needs to be developed.

The priority mechanism presented in Section 4.4 strongly reduces the latency of the frame to be decoded. In the original 3D-Wave implementation, the latency of the first frame is $58.5 \mathrm{~ms}$, using the FHD Rush_Hour sequence with 16 cores. Using the frame scheduling policy, the latency drops to $15.1 \mathrm{~ms}$. This latency is further reduced to $9.2 \mathrm{~ms}$ when the priority policy is applied together with frame scheduling. This is almost the same as the latency of the 2D-Wave, which decodes frames one-by-one. Figure 7(b) depicts the number of MBs processed per $m s$ when this feature is used.
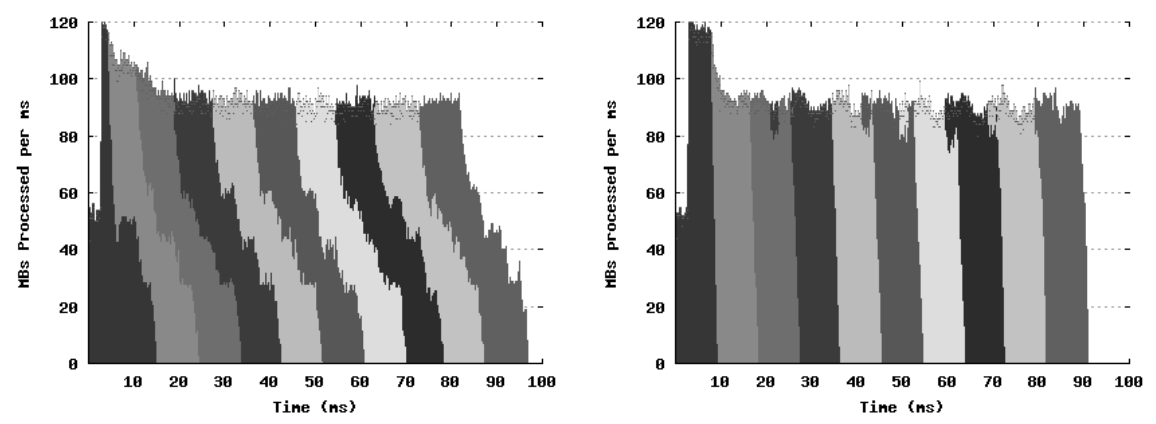

(a) Number of MBs processed per ms us- (b) Number of MBs processed per ms using frame scheduling and frames to which ing frame scheduling and the priority polthese MBs belong. icy.

Fig. 7. Results for frame scheduling and priority policy for FHD Rush_Hour in a 16-core processor. Different colors represent different frames.

Different scenarios were used to analyze the impact of frame scheduling and priority on scalability. The chosen scenario uses 3 and 6 frames in flight, with and without Frame Priority. Figures 8(a) and 8(b) depict the impact of the presented techniques on the scalability. 2D-Wave (2DW) and 3D-Wave (3DW) scalability results are presented as guidelines. In Fig. 8, FS refers to the frame scheduling. The addition of Frame Priority has no significant impact on speedup, thus it is not shown in the figure. The reported scalability is based on the 2D-Wave execution time in a single core.

As can be seen in Fig. 8(a), 6 frames in flight are not enough to leverage the scalability for 64 cores when decoding a SD sequence. The maximum speedup of 23 is result of the relatively low MB parallelism of SD frames. As presented in Table ??, the 2D-Wave for SD has a maximum speedup of 8 . For the HD sequence (figure not shown) 6 frames in flight are already close to the original 3D-Wave scalability. The maximum speedups are 24 and 45, for three and six frames in 


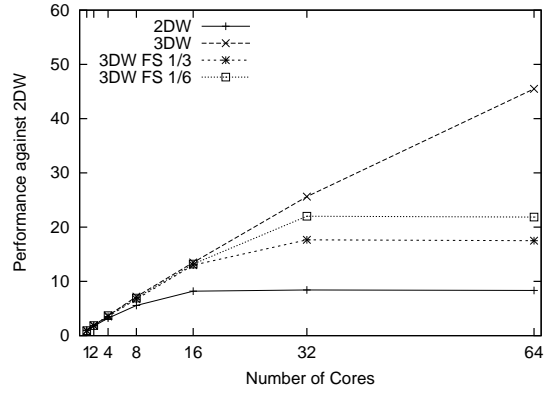

(a) Scalability SD

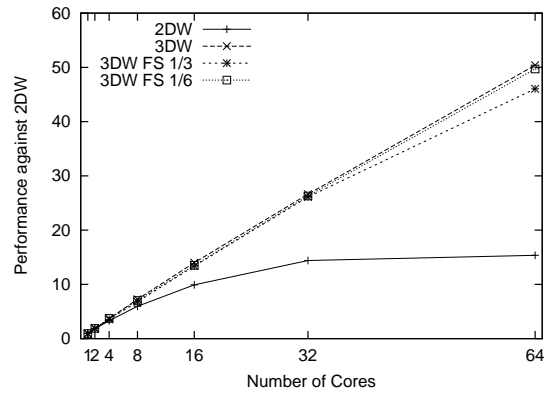

(b) Scalability FHD

Fig. 8. Frame Scheduling and Priority Scalability Results for Rush Hour 25 Frames

flight, respectively. The latter is $92 \%$ of the maximum 3D-Wave speedup. For the FHD resolution, depicted in Fig. 8(b), three frames in flight provide a speedup of 46. When 6 frames are used the difference between the FS and the original $3 \mathrm{D}$-Wave implementation is only $1 \%$.

The FS and Priority policies are efficient on reducing the number of frames in flight and their latency. The effects on scalability of FS depends on the intraframe parallelism and the allowed number of frames in flight. Priority has a negligible effect on scalability.

\subsection{Bandwidth requirements}

In this section, the intra-chip bandwidth requirements for the 3D-Wave and its Frame Scheduling and Priority policies are reported. The data traffic between level 2 and level 1 data caches are measured. The access to main memory is not reported by the simulator. The effects of these techniques on data traffic between L2 and L1 data caches are depicted in Fig. 9(a) and 9(b). The figures depict the required data traffic for SD and FHD resolutions, respectively, from 1 to 64 cores. 2D-Wave (2DW) and 3D-Wave (3DW) data traffic results are presented as comparison. In the figures, FS refers to the frame scheduling while $\mathrm{P}$ refers to the use of frame priority.

Data locality decreases as the number of cores increases, because the task scheduler does not take into account data locality when assigning a task to a core (except with the tail submit strategy). This decrease in the locality leads to cache thrashing, that also contributes to traffic increase. Due to these effects, the 3D-Wave increases the data traffic by approximately $104 \%, 82 \%$, and $68 \%$ when going from 1 to 64 cores, for SD, HD, and FHD, respectively.

The original 3D-Wave requires the least communication between L2 and L1 data caches for 8 cores or more. It is approximately $20 \%$ to $30 \%$ (from SD to FHD) more data traffic efficient than the original 2D-Wave, for 16 cores or more. This is caused by the high data locality of the original 3D-Wave technique. 


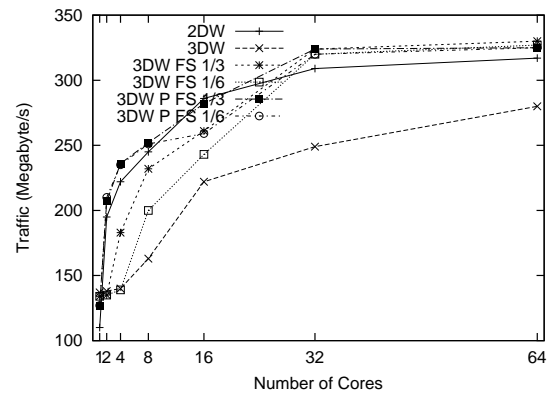

(a) SD

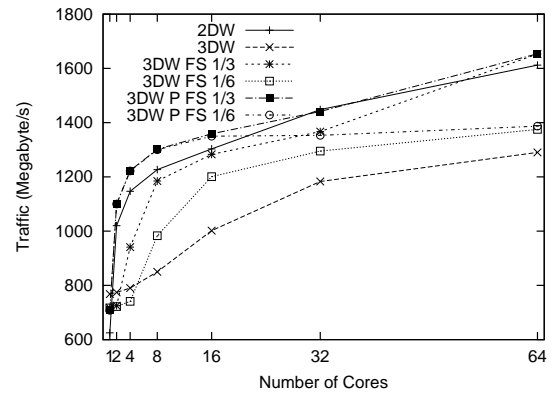

(b) FHD

Fig. 9. Frame Scheduling and Priority Data Communication Results for Rush Hour 25 Frames

The 3D-Wave implementation fires new frames as soon as their dependencies are met. This increases the probability of the reference areas of a MB to be present in the system. The probability increases because nearby area of several frames are decoded together, so the reference area is still present in data caches of other cores. This reduces the data traffic because the motion compensation (inter-frame dependency) requires a significant portion of data to be copied from previous frames.

The use of FS and Priority has a negative impact on the L2 to L1 data cache traffic. The use of FS and Priority decreases the data locality, as they increase the time between processing MBs from co-located areas of consecutive frames. However, when the number of frames are enough to sustain a good scalability, the increased data traffic when using FS and Priority is still lower than the data traffic of 2D-Wave implementation. For SD, the data traffic for FS and Priority is higher than the 2D-Wave when the available parallelism is not enough to leverage for 32 and 64 cores. The same happens for the HD using only 3 frames in flight. For FHD, 2D-Wave is the technique that requires most data traffic, together with FS for 3 frames in flight. When the number of frames in flight are enough to leverage to 32 or 64 cores, FS is 4 to $12 \%$ more efficient than 2D-Wave. FS and Priority can be 3 to $6 \%$ data traffic less efficient than 2D-Wave in the cases when number of frames in flight are insufficient to leverage to the number of cores.

With the data traffic results it is possible to calculate the L2 to L1 bandwidth requirements. The bandwidth is calculated by dividing the total traffic by the time to decode the sequence in seconds. The total amount of intra chip bandwidth required for 64 cores is $21 \mathrm{~GB} / \mathrm{s}$ for all resolutions of Rush_Hour sequence. The bandwidth is independent of the resolution because the number of MBs decoded per time unit per core is the same. 


\subsection{Impact of the Memory Latency}

The type of interconnection used and the number of cores in the system can contribute to increase the memory latency. Data become scattered with the increasing number of cores and this increases the length of the path where the data have to travel from where they are stored to where they are needed inside the chip. In this section, the effects of an higher L2 to L1 data cache average latency is analyzed. To study these effects one second (25 frames) of the Rush Hour sequence where decoded using several average memory latencies on the three available resolutions.

In the previous experiments, the average memory L2 data cache latency was set to be 40 cycles. The simulation does not include the communication between L1 and L2. Instead an average memory latency is set to delay the access to each data request. Each sequence was decoded with the average memory latency (AML) set to a value varying from 40 (original case) to 100 cycles, with increments of 10. Figure 10(a) depicts the scalability results for the FHD resolution. Scalability results are relative to the original 40 cycles AML running on a single core.

The results show that memory latency does not drastically affects the scalability. The scalability decreases by $10 \%$ when the AML goes from 40 to 100 cycles on 64 cores. However, not only scalability is an issue on multicore systems. To reflect the impact of the increased memory latency on performance Figure 10(b) depicts the performance scalability using the single core 40 cycles AML performance as baseline for the performance scalability.

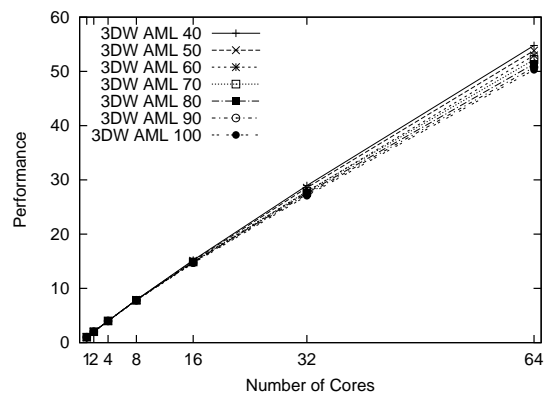

(a) Scalability

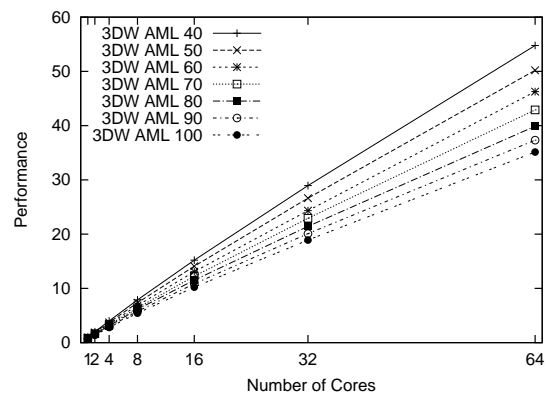

(b) Performance

Fig. 10. Memory Latency Scalability and Performance Compared with Single Core AML 40 Results for Rush Hour FHD 25 Frames

When the overhead of memory latency is combined with its effects on scalability it drastically decreases the system's performance. For a large number of cores, such as 32 and 64, this decrease in performance can make a large system infeasible. This can be seen in Fig. 10(b) where the 64 cores system with 
increased AML has almost the same performance as a 32 cores system with a smaller AML.

\subsection{L1 Cache Size Impact on Scalability}

Memory hierarchy has an increasing impact on performance. To investigated the benefits of intra-core memory, we analyzed the influence of the amount of L1 data cache memory per core on the scalability and bandwidth requirements. The simulated amount of L1 cache was 16, 32, 64, 128, and 256KB. The original $64 \mathrm{Kbyte}$ data-cache has 64-byte lines and is 4-way set-associative with LRU replacement and write allocate. The only parameter changed on the simulations was the number of sets. The Performance results are depicted in Figure 11(a) for FHD resolution. Performance results are based on the decoding time on a single core with the original $64 \mathrm{~KB}$ cache. The $16 \mathrm{~KB}$ cache has a performance drop of $45 \%$ and $48 \%$ for 1 and 64 cores, respectively. The $32 \mathrm{~KB}$ cache has a performance drop of approximately $30 \%$ for any number of cores. The reason of this performance drop is depicted in Figure 11(b), which presents the L1L2 cache data traffic for FHD resolution. The traffic is directly related to the number of cache misses, as new data have to be copied from L2 to L1. Also, misses generate write back to L2 when the cache is fully utilized.

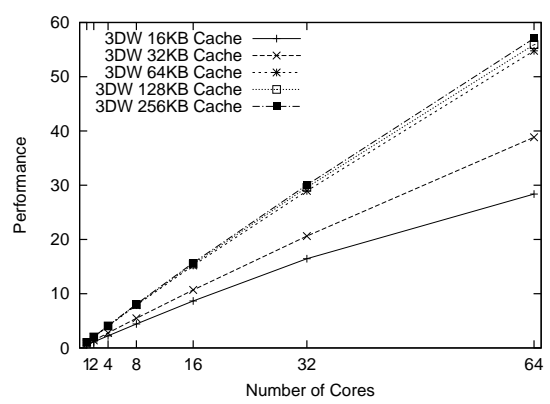

(a) Scalability

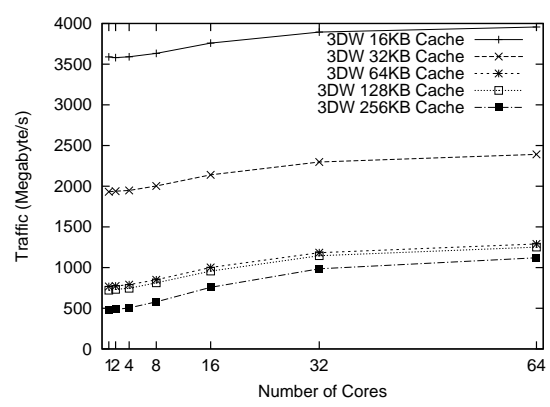

(b) Bandwidth

Fig. 11. Cache Size Influence on Performance and Traffic Results for Rush Hour FHD 25 Frames

The high performance drop of the FHD for $16 \mathrm{~KB}$ and $32 \mathrm{~KB}$ caches can be seen in the traffic results. The traffic increase is 4.7 times the baseline for one core and 3.1 times for 64 cores for $16 \mathrm{~KB}$ cache and 2.5 to 1.8 times for $32 \mathrm{~KB}$ cache, for 1 and 64 cores, respectively. For SD 16KB cache, the increase of traffic goes from $66 \%$ for 1 core to $19 \%$ for 64 cores, when compared with the reference $64 \mathrm{~KB}$ cache. For HD these numbers go from $72 \%$ to $26 \%$. For $32 \mathrm{~KB}$ caches the range is shorter going from a decrease of $7 \%$ for 1 core to an increase of $8 \%$ for 64 cores for SD and for HD these results go from $12 \%$ to $5 \%$ increase, respectively. 
A MB line for FHD resolution occupies $45 \mathrm{~KB}$, while an $\mathrm{HD} 30 \mathrm{~KB}$, and an $\mathrm{SD} 17 \mathrm{~KB}$. The caches should be able to store more than one MB line as it is reused on the decoding process of the same frame and serves as input to motion compensation of the other frames. For FHD, the small caches require the data to be reloaded many times. This explains the heavy thrashing of the FHD, while for the other resolutions the impact is not so high.

The size of a MB line also explains the small increase in performance for caches larger than $64 \mathrm{~KB}$. Once the dataset fits on the cache the application behaves like a memory stream application and makes no use of the extra space. This small increase in performance resulted from the increased data cache is also reflected on the data traffic. For all resolutions the change in data traffic ranges from -10 to $5 \%$, approximately, except for the $256 \mathrm{~KB}$ cache for FHD. The $256 \mathrm{~KB}$ cache for FHD has a decrease in traffic from 27 to $13 \%$, from 1 to 64 cores. Even with this high reduction in traffic the performance gain is no more than $4 \%$. This gain is similar to the other resolutions with a less significant decrease and sometimes with an increasing in traffic.

\subsection{Influence of the Parallelization Support Over Scalability}

Alvarez et al. [16] implemented the 2D-Wave approach on an architecture with 64 dual core IA-64 processors. Each one of the 128 cores works at 1,6 GHz, with a $8 \mathrm{MB}$ L3 cache and $533 \mathrm{MHz}$ Bus, and the system has a total $512 \mathrm{~GB}$ RAM. Their results show that the scalability is severely limited by the thread synchronization and management overhead, i.e., the time it takes to submit/retrieve a task to/from the task pool. On their platform it takes approximately XXX times as long to submit/retrieve a task as it takes to decode a MB. To analyze the impact of the TLP overhead on the scalability of the 3D-Wave, we replicate this TLP overhead by increasing the Thread Pool Library by adding dummy calculation.

The inserted extra overheads are 10\%, 20\%, 30\%, 40\%, 50\%, and $100 \%$ of the average MB decoding time, which is 4900 cycles. Because of the Tail Submit enhancement not every MB requests or submits a task to the Thread Pool. This causes a total performance overhead of only $3 \%$ for a single core when comparing the $100 \%$ TPL overhead against the baseline 3D-Wave. The effects of this increased overhead is depicted on Figures 12(a), and 12(b), for SD and FHD resolutions, respectively.

The results for SD resolution show the impact of the increase overhead on the scalability. For 32 cores the scalability is considerably reduced when the overhead is $40 \%$ or more. For 64 cores the effects of the extra overhead reduces the scalability. The SD resolution is very affected with the increased overhead because the intra frame resolution is comparatively low and the lines are short, which increases task submition per frame. For the HD resolution (figure not shown) the increase in overhead limits the scalability to 32 cores while for FHD it slows down the scalability, but does not limit it. As the resolution increases the requests to TPL per MB ratio decreases and so the impact of the extra overhead. 


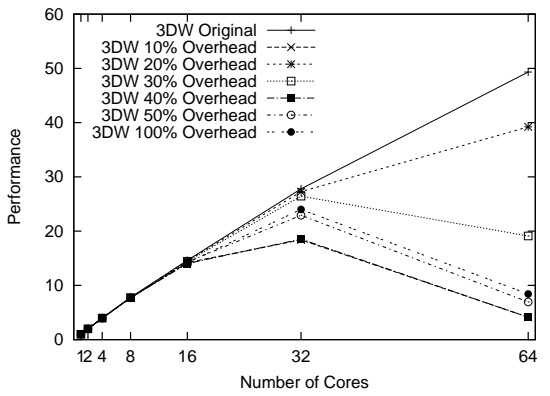

(a) $\mathrm{SD}$

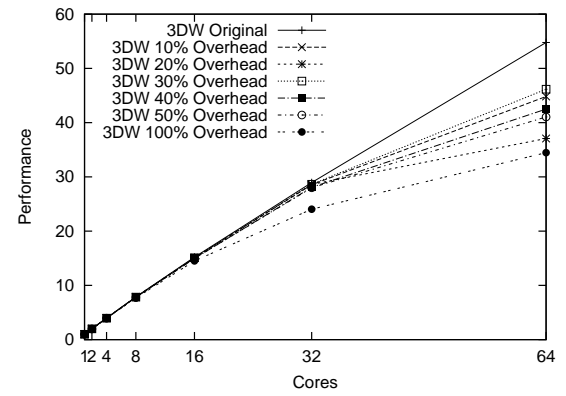

(b) FHD

Fig. 12. TPL Overhead Effects on Scalability for Rush Hour Frames

These results show the drastic effects of the overhead on the scalability, even with enhancements that reduces the requests to the parallelization support.

\subsection{CABAC Influence}

In this work $\mathrm{CABAC}$ is considered to be handle by a specific accelerator. In this section we evaluate the requirements of this accelerator to allow the presented scalability results of the 3D-Wave. Figure 13 depicts the maximum speedup that can be achieved for H.264 decoding for different numbers of MB processing cores by different speeds of the CABAC accelerators. The baseline CABAC accelerator is considered to have the same performance as the TM3270 Trimedia processor. The CABAC accelerators perform entropy decoding while the other cores perform macroblock (MB) decoding. These results were obtained using a trace-driven, abstract-level simulator that calculates the critical path length given the $\mathrm{CABAC}$ and $\mathrm{MB}$ dependencies and the $\mathrm{CABAC}$ and $\mathrm{MB}$ processing times. Traces have been obtained using the simulator of a many-core of TM3270 Trimedia processors.

The results show that if $\mathrm{CABAC}$ decoding is not accelerated, then the speedup is limited to 7.5 .

The reason why it is more scalable to accelerate CABAC than it is to employ more CABAC processors is that more MBs can be processed in parallel since more thread-level parallelism is exposed. These results show that the CABAC processing does not impose a limitation to the 3D-Wave technique.

\section{Conclusions}

In this paper, an implementation of the 3D-Wave parallelization technique on an embedded CMP has been presented and evaluated. The implementation requires to identify intra-frame MB dependencies dynamically, which led to the development of a mechanism where MBs subscribe themselves to the MBs in the 


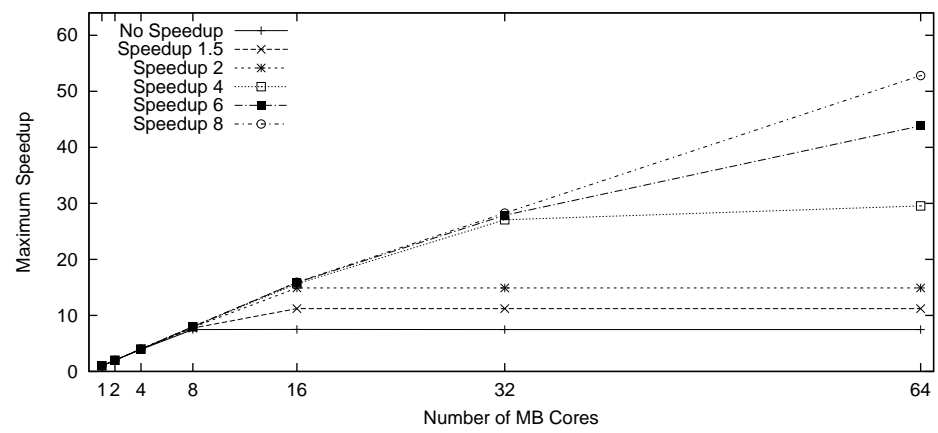

Fig. 13. Maximum Scalability per CABAC processor and Accelerators

reference areas they depend upon. We have also presented policies for reducing the number of frames in flight and the frame latency.

The results show that the 3D-Wave implementation can leverage a multicore system with up to 64 cores. While the 2D-Wave has a speedup about 8 for 16 cores or more, for SD resolution, the 3D-Wave has a speedup of almost 45 on 64 cores. These results were achieved for sequences with no frame-, slice- or GOP-level parallelism.

Frame Scheduling and Priority policies to reduce frames in flight and frame latency were presented and analyzed. These policies are efficient with minimum impact in performance. Memory latency, L1 cache size, and parallelization overhead effects on scalability and performance were also presented. CABAC accelerator performance requirement for supporting 3D-Wave scalability is presented.

Future work includes the development of an automatic frame scheduling technique, the implementation of the 3D-Wave on general purpose processors, and the implementation of the 3D-Wave in the encoder. A 3D-Wave implementation of the encoder can be applied for high definition, low latency encoding on multi-processors.

\section{Acknowledgment}

This work was partially supported by the European Commission in the context of the SARC integrated project \#27648 (FP6), the Ministry of Science of Spain and European Union (FEDER funds) under contract TIC-2004-07739-C02-01, and the European Network of Excellence on High-Performance Embedded Architecture and Compilation (HiPEAC). The authors would like to thank Anirban Lahiri from NXP for his collaboration on the experiments.

\section{References}

1. Okano, F. and Kanazawa, M. and Mitani, K. and Hamasaki, K. and Sugawara, M. and Seino, M. and Mochimaru, A. and Doi, K.: Ultrahigh-Definition Television 
System With 4000 Scanning Lines. In: NAB Broadcast Engineering Conference proceedings. (2004) 437-440

2. Drose, M., Clemens, C., Sikora, T.: Extending single-view scalable video coding to multi-view based on h. 264/avc. In: Image Processing, 2006 IEEE International Conference on. (2006) 2977-2980

3. Meenderinck, C., Azevedo, A., Alvarez, M., Juurlink, B., Ramirez, A.: Parallel scalability of h.264. In: Proceedings of the first Workshop on Programmability Issues for Multi-Core Computers. (January 2008)

4. Meenderinck, C., Azevedo, A., Juurlink, B., Alvarez, M., Ramirez, A.: Parallel Scalability of Video Decoders. Journal of Signal Processing Systems (August 2008)

5. Rodriguez, A., Gonzalez, A., Malumbres, M.P.: Hierarchical parallelization of an h.264/avc video encoder. In: Proc. Int. Symp. on Parallel Computing in Electrical Engineering. (2006) 363-368

6. Chen, Y., Li, E., Zhou, X., Ge, S.: Implementation of H. 264 Encoder and Decoder on Personal Computers. Journal of Visual Communications and Image Representation 17 (2006)

7. van der Tol, E., Jaspers, E., Gelderblom, R.: Mapping of H.264 Decoding on a Multiprocessor Architecture. In: Proc. SPIE Conf. on Image and Video Communications and Processing. (2003)

8. : International Standard of Joint Video Specification (ITU-T Rec. H. 264ISO/IEC 14496-10 AVC) (2005)

9. Oelbaum, T., Baroncini, V., Tan, T., Fenimore, C.: Subjective Quality Assessment of the Emerging AVC/H.264 Video Coding Standard. In: Int. Broadcast Conference (IBC). (2004)

10. Alvarez, M., Salami, E., Ramirez, A., Valero, M.: A Performance Characterization of High Definition Digital Video Decoding using H.264/AVC. In: Proc. IEEE Int. Workload Characterization Symposium. (2005) 24-33

11. Ostermann, J., Bormans, J., List, P., Marpe, D., Narroschke, M., Pereira, F., Stockhammer, T., Wedi, T.: Video Coding with H.264/AVC: Tools, Performance, and Complexity. IEEE Circuits and Systems Magazine 4(1) (2004) 7-28

12. van de Waerdt, J., Vassiliadis, S., Das, S., Mirolo, S., Yen, C., Zhong, B., Basto, C., van Itegem, J., Amirtharaj, D., Kalra, K., et al.: The tm3270 media-processor. In: MICRO 05: Proceedings of the 38th International Symposium on Microarchitecture. (November 2005) 331-342

13. : X264. A Free H.264/AVC Encoder

14. Alvarez, M., Salami, E., Ramirez, A., Valero, M.: HD-VideoBench: A Benchmark for Evaluating High Definition Digital Video Applications. In: IEEE Int. Symp. on Workload Characterization. (2007)

15. Hoogerbrugge, J., Terechko, A.: A Multithreaded Multicore System for Embedded Media Processing. (To Appear) Transactions on High-Performance Embedded Architectures and Compilers 4(2) (2009)

16. Alvarez, M., Ramirez, A., Valero, M., Meenderinck, C., Azevedo, A., Juurlink, B.: Performance evaluation of macroblock-level parallelization of h.264 decoding on a cc-numa multiprocessor architecture. In: Proceedings of the 4CCC: 4th Colombian Computing Conference. (April 2009) 